\title{
GENERACIÓN DE EMPLEO EN \\ LA CONSTRUCCIÓN: EL CASO DE UNA VIVIENDA MÍNIMA EN MÉXICO
}

\author{
Christian Araud ** \\ CIMAS, Universidad Nacional Autónoma de México
}

EN UN SISTEMA económico-social en donde predomina la libre empresa, los dirigentes tienen como principal objetivo aumentar sus ganancias tanto como sea posible, sin reparar en las consecuencias sociales que pueda tener como efecto la acción misma de producir. Esto oeasiona que la mayoría de los criterios que se han desarrollado para la evaluación de inversiones estén basados en la rentabilidad. Por lo tanto, aun para el estado, al que por principio se puede suponer interesado en atacar problemas como el de la desequilibrada distribución del ingreso y el del desempleo, es difícil encontrar criterios adecuados, desde el punto de vista social, para la elección de inversiones. Esto señala la necesidad de que la investigación económica se enfoque a la búsqueda de indicadores que permitan juzgar adecuadamente una inversión en cuanto a sus implicaciones sociales.

Uno de los aspectos sobresalientes, que ayudan a conocer las consecuencias sociales de la producción, es el que se refiere al empleo que se genera en la elaboración de un producto, o sea, el trabajo contenido en él. En este trabajo se plantea el problema de la medición de dicho empleo, llevando a cabo el cálculo para el caso de una vivienda mínima en México.

En primer lugar, se exponen algunas ideas generales del análisis de insumo-producto y la forma en que se utiliza para nuestro propósito. Posteriormente, a partir de este método se deriva cierto número de "coeficientes de empleo" para los insumos del sector de la construcción, y por último se aplican esos coeficientes a una vivienda.

\section{Metodología}

Para resolver el problema de la medición del empleo incluido en cualquier producto, se utilizan las matrices de insumo-producto y una

* Este trabajo fue elaborado con base en los estudios que forman parte del proyecto de investigación sobre empleo y construcción de vivienda en México, llevado a cabo por el Centro de Estudios Econónicos y Demográficos de El Colegio de México en colaboración con el Centro de Desarrollo de la ocDe de París, durante 1971-1973.

${ }_{* *}^{*}$ Agradezco a Santiago Rincón Gallardo, del Centro de Investigación en Matemáticas Aplicadas y Sistemas (cimAs), su valiosa colaboración en este trabajo. 
metodología derivada del modelo de Leontief. Sin embargo, una cuestión que dificulta nuestras estimaciones es el hecho de que el monto de la producción de viviendas aparece en la matriz de insumo-producto como un bien de demanda final junto con diversos productos del sector "construcción" tales como carreteras, puentes, presas y toda clase de edificios. ${ }^{1}$ Por este motivo y dado que se pretende estimar el empleo requerido para la construcción de una vivienda, es necesario estimar una función producción para el producto vivienda con el detalle adecuado, como se indica a continuación.

Para construir 1000 metros cuadrados de vivienda, se requieren:

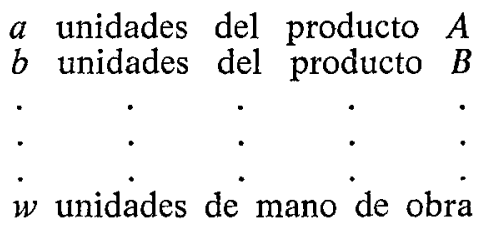

y a partir de aquí convertir las cantidades de productos $A, B$, en unidades de trabajo.

Una manera de hacer esta conversión sería hacer el análisis para cada producto $A, B$, etc., y estimar otras funciones de producción que expresen los insumos necesarios para construir una unidad del producto $A$ :

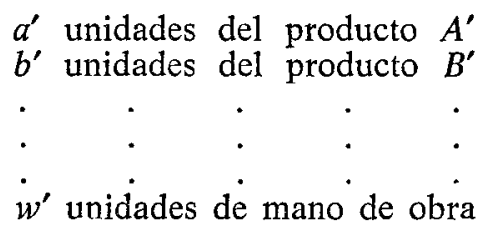

y continuar así en forma sucesiva hasta que el residuo sea tan pequeño que se pueda despreciar. Sin embargo, tal procedimiento implicaría un trabajo muy laborioso de recolección de información, por lo que es necesario utilizar un método más accesible.

Debido a la diversidad de productos específicos que intervienen en el proceso de construcción de una vivienda, por conveniencia, se han formado 'grupos en los que aparecen las distintas variedades de un mismo bien ( $v$. gr. para el caso del cemento se agrupan en un solo rubro todas las clases utilizadas) expresadas en términos monetarios en un momento dado, y después se estiman "coeficientes" que expresen la cantidad $x$ de trabajo en un año (en promedio), que se necesitan para producir \$1000000 de “cemento" en 1965.

Una vez estimados tales coeficientes para los principales productos que "entran" como insumos intermedios de la vivienda, podemos calcular con relativa facilidad el empleo generado.

1 Una explicación detallada acerca de este problema aparece en R. Stone Comp., Input-Output Relationships, 1954-1966, Londres, Chapman and Hall, 1963, Cap. II. 
En efecto, si el precio de una vivienda expresado en $x$ cantidad de dinero por metro cuadrado se presenta como los presupuestos que se elaboran en los proyectos de construcción:

$$
\begin{aligned}
& x_{1}=\text { Producto } 1 \\
& \cdot \\
& \dot{x}=\text { Producto } n \\
& \dot{x}_{n}=\text { Salarios brutos } \\
& Y=\text { Impuestos y utilidades }
\end{aligned}
$$

y si tenemos que:

$$
\begin{aligned}
& c_{1}=\text { mano de obra por } \$ 1 \text { del producto } 1 \\
& \dot{\cdot} \\
& \dot{c_{n}}=\text { mano de obra por } \$ 1 \text { del producto } n \\
& m=\text { salario promedio anual }
\end{aligned}
$$

Entonces, el número de personas empleadas será:

$$
N=\sum_{i} c_{i} x_{i}+S / m
$$

en donde $S / m$ es el empleo directo (empleo en la obra) y $\sum_{i} c_{i} x_{i}$ el empleo indirecto (empleo incorporado en los insumos).

Sin embargo, la frontera entre empleo directo e indirecto es un poco flexible dado que la función producción utilizada, la de la empresa que construye la vivienda, puede variar para el mismo producto según el nivel de integración de la empresa. ${ }^{2}$

\section{LA MATRIZ DE INSUMO-PRODUCTO}

En el análisis de insumo-producto se considera la economía diviđida en sectores de origen y sectores de destino y las relaciones entre ellos son expresadas en dos cuentas fundamentales:

$$
\begin{aligned}
& X_{i}=\sum_{i} X_{i j}+M_{j}+V_{i}+A_{i} \\
& X_{i}=\sum_{i} X_{i j}+D_{i}
\end{aligned}
$$

en donde:

$$
\begin{aligned}
X_{j}= & \text { producción bruta de la rama } j \\
X_{i j}= & \text { consumo intermedio de producto } i \text { por la rama } i \text { (o consumo } \\
& \text { del sector } j \text { que tiene su origen en el sector } i \text { ) } \\
M_{i}= & \text { importaciones de la rama } j
\end{aligned}
$$

2 A veces, se subcontrata la elaboración de productos terminados que pueden ser elaborados por el personal de la empresa como en el caso de la herrería. 
$V_{j}=$ valor agregado neto de la rama $j$

$A_{j}=$ depreciación de la rama $j ; \mathrm{y}$

$D_{i}=$ demanda final del producto $i$

Si se conoce el número de personas que trabajan en la rama $j$, que denotaremos $N_{j}$, y que según las cuentas nacionales participan en la producción bruta $X_{j}$ durante el período que coincide con el de las cuentas, y si denotamos por $Z_{j}$ el número de personas que trabajan en todas las ramas para producir $X_{j}$, producto de la rama $j$, la expresión de los coeficientes que interesan para nuestros cálculos sería:

$$
z_{i}=Z_{j} / X_{j}
$$

Este coeficiente debe considerarse como una aproximación dado que representa el flujo de los empleos requeridos durante un año para la producción agregada y no la suma exacta de todos los empleos requeridos para un producto específico.

III. Modelo de Magaud ${ }^{3}$

Sea el empleo por unidad de producción bruta en la rama $j$ :

$$
n_{i}=N_{j} / X_{i}
$$

y sea $T_{j}$ el empleo que sería necesario en la rama $j$ para producir $X_{j}$ si los insumos hubieran sido abastecidos al principio el período considerado.

Para estimar $z_{j}$, el empleo por unidad de producción bruta para el producto $j$, haremos el balance del empleo incluido en la producción del sector $j$. Por un lado tendríamos $Z_{j}=z_{j} X_{i}$ (por definición) y por otro $E_{i j}$, el empleo incluido en el consumo intermedio del producto $i$ en la rama $j$, para cada rama, y $E^{\prime}$; el empleo incluido en la depreciación de capital y $T_{i}$ que ya fue definido.

Tendremos entonces:

$$
Z_{j}=\sum_{i} E_{i j}+E_{j}^{\prime}+T_{i}
$$

y de acuerdo con la hipótesis fundamental de Leontief (rendimientos constantes) :

$$
\begin{aligned}
& a_{i j}=X_{i j} / X_{j} \\
& E_{i j}=z_{i} X_{i j}=z_{i}\left(X_{i j} / X_{j}\right) X_{j}=z_{i} a_{i j} X_{j}
\end{aligned}
$$

Si establecemos además la hipótesis de que el desglose de la depreciación $A_{j}$, para los distintos productos es idéntico al de las inversiones de la rama $j$, tendremos:

$$
A_{j}=X_{j} \sum_{i} b_{i j}+J_{j}^{\prime}
$$

3 Jacques Magaud, "Equivalent travail d'une production", Population, París, agosto de 1967, pp. 53-69. 
en donde $J_{j}^{\prime}=$ depreciación de las importaciones productivas de la rama $j, \mathrm{y} b_{i j}=1$ la depreciación por origen $\mathrm{y}$ destino, hecha proporcional al volumen de producción del sector de destino $\left(X_{i}\right)$

Por lo tanto:

$$
E_{i}^{\prime}=X_{j} \underset{i}{\sum} b_{i j} z_{i}
$$

Entonces, al sustituir en (3), se tiene

$$
Z_{j}=X_{j} \sum_{i}\left(a_{i j}+b_{i j}\right) z_{i}+T_{j}
$$

y dado que $t_{j}=T_{j} / X_{j}$, entonces:

$$
z_{j}=\sum_{i}\left(a_{i j}+b_{i j}\right) z_{i}+t_{j}
$$

Ahora bien, si denotamos $C$ como la matriz tal que ${ }^{4}$

$$
C=\left[c_{i j}\right]=[I-A-B]^{-1}
$$

en donde

$$
I=\text { Matriz unitaria; } A=\left[a_{i j}\right] \text { y; } B=\left[b_{i j}\right]
$$

entonces

$$
z_{j}=\sum_{i} c_{i j} t_{i}
$$

Ahora bien, si multiplicamos $c_{i j}$ primero por $t_{i}$ y después por $X_{j}$, tenemos la matriz:

$$
m_{i j}=X_{j} c_{i j} t_{i}
$$

que expresa el empleo en la producción bruta del bien $i$, que tiene su origen en la rama $i$.

Sumando sobre los sectores le origen se obtiene $Z_{j}$, el empleo por producto:

$$
Z_{j}=\sum_{i} m_{i j}
$$

y haciendo la suma sobre los sectores de destino, tendremos $N_{i}$, el empleo por rama:

$$
N_{i}=\sum_{i} m_{i j}
$$

y el empleo total en la economía será entonces:

$$
W=\sum_{j} Z_{j}=\sum_{i} N_{i}=\sum_{i j} m_{i j}
$$

4 La matriz de coeficeintes técnicos $A$ no debe confundirse con los vectores $A$. referentes a la depreciación sectorial. 
De la ecuación (4) se puede calcular $t_{i}$ :

$$
N_{i}=\sum_{i} c_{i j} t_{i} X_{i}=t_{i} \sum_{j} c_{i j} X_{i}
$$

y sea

$$
t_{i}=n_{i} / m_{i}
$$

en donde:

$$
\left.m_{i}=\underset{j}{\left(\Sigma_{i j}\right.} c_{i j}\right) / X_{i}
$$

$m_{i}$ aparece como un coeficiente multiplicador de empleo.

Una vez establecido el proceso de cálculo para el empleo generado en la producción se exponen dos resultados de su aplicación.

La estimación de los coeficientes señalados se hizo tomando como base el cuadro de insumo-producto de México elaborado por el Banco de México para 1965, con los resultados que aparecen en el cuadro 1.

\begin{tabular}{|c|c|c|c|c|}
\hline & Total & Obreros & Empleados & Profosionistes \\
\hline $\begin{array}{l}\text { Agricul tura } \\
\text { Minerfa } \\
\text { Patróleo } \\
\text { Alimentos } \\
\text { Textiles } \\
\text { Madera } \\
\text { Qufmica } \\
\text { Minerales no metálicos } \\
\text { lietálicos básicos } \\
\text { irecánica } \\
\text { Construoción } \\
\text { Electrioidad } \\
\text { Comercio } \\
\text { Transporto } \\
\text { Servicios }\end{array}$ & $\begin{array}{r}100.6 \\
20.1 \\
9.5 \\
55.3 \\
41.9 \\
28.9 \\
23.4 \\
20.6 \\
14.8 \\
20.3 \\
29.2 \\
13.0 \\
10.5 \\
31.4 \\
35.9\end{array}$ & $\begin{array}{r}98.7 \\
16.0 \\
7.3 \\
51.4 \\
3 \varepsilon .5 \\
25.6 \\
18.3 \\
18.1 \\
11.7 \\
16.9 \\
25.5 \\
8.7 \\
13.5 \\
24.4 \\
20.8\end{array}$ & $\begin{array}{l}1.4 \\
2.6 \\
1.5 \\
3.0 \\
2.5 \\
2.2 \\
3.7 \\
1.6 \\
2.0 \\
2.3 \\
2.2 \\
3.3 \\
2.5 \\
6.1 \\
8.5\end{array}$ & $\begin{array}{l}0.5 \\
1.5 \\
0.7 \\
0.8 \\
0.9 \\
1.0 \\
1.4 \\
0.9 \\
1.0 \\
1.1 \\
1.5 \\
1.0 \\
0.6 \\
1.0 \\
6.6\end{array}$ \\
\hline
\end{tabular}

Cuadro 1

México: Coeficientes de empleo para 15 sectores por categorías a

a Necesarios para obtener una producción de $\$ 1000000$.

El sector "construcción" junto con el de "alimentos" aparece como uno de los menos integrados. Lo contrario se observa en los sectores "petróleo" y "minerales" que aparecen con un alto grado de integración.

La comparación de los coeficientes de empleo por producto y por rama de actividad permite distinguir tres aspectos relevantes:

a) Un margen de variación de los coeficientes de empleo por ramas de actividad muy grande: de 6.8 en la rama "petróleo" hasta 192.9 la "agricultura".

Haciendo abstracción de la deficiencia de los datos, esto se debe con toda seguridad, a que los empleados en el sector petróleo, altamente mecanizado y con tecnología avanzada, tienen una productividad mucho más alta y a que trabajan más tiempo en un año que los trabajadores agrícolas, en donde prevalece un alto nivel de subocupación y desocupación. 
b) La amplitud de la variación disminuye cuando consideramos los coeficientes específicos para productos: en este caso la variación entre los mismos sectores señalados, va desde 9.5 para el producto "petróleo" hasta 100.6 para el producto "agricultura".

c) El sector "alimentos" registra el coeficiente más reducido, lo que refleja la alta productividad a que opera. En cambio, los productos alimenticios aparecen con un coeficiente bastante elevado debido con toda seguridad a que incluye una gran cantidad de productos del campo y en consecuencia contiene un volumen elevado de mano de obra campesina.

\section{LAS CATEgoRías PROFESIONALES}

El análisis del empleo generado se hace a través de cinco categorías profesionales:

a) campesinos

b) obreros

c) empleados

d) técnicos

e) profesionistas y ejecutivos

Si se denota por $N_{i k}$ la población activa en la rama $i$ para la categoría $k$ podemos escribir:

1) $n_{i k}=N_{i k} / X_{i}$, como el empleo unitario en la rama $i$ y la categoría $k$.

2) $t_{i k}=n_{i k} / m_{i}$, el empleo directo unitario en la rama $i$ y la categoría $k$.

3) $z_{j k}=\sum_{i} c_{i j} t_{i k}$, el coeficiente de empleo de la categoría $k$ y el producto $j$.

Utilizando las estimaciones sobre empleo realizados por Keesing y Manne ${ }^{5}$ para 1960 y con una tasa dada de crecimiento tendremos una proyección para 1965 para 15 ramas y 3 categorías (agrupando las dos primeras categorías, $a$ y $b$ y las dos últimas $d$ y $e$ en una sola respectivamente), se obtienen los resultados que aparecen en el cuadro 1.

El gran volumen de fuerza de trabajo y la baja productividad del campo explican la importancia de los productos de las ramas agrícola, alimenticia, de textiles y de madera, respecto al número de obreros (sobre todo de la primera). En cambio, en la producción de la agricultura aparece el más bajo índice de utilización de profesionistas.

\section{EMPLEO GENERADO EN 16 MATERIALES DE CONSTRUCCIÓN}

Los coeficientes que acabamos de calcular se refieren al producto de la rama, el cual es completamente distinto al de los productos específicos

5 Donald B. Keesing y Alan S. Manne, "Memorándum sobre el modelo DINAMICO" (Mimeo.), México, Banco de México, 1970, cuadro 6. 
que la constituyen en la realidad, ${ }^{6}$ sobre todo cuando se conşidera el valor de la producción bruta de un sector y el valor a los precios de mercado de los productos específicos. La situación ideal sería disponer de una matriz con un número de ramas igual al de productos existentes, pero para México se dispone sólo de un cuadro de 45 sectores para 1965 que se utiliza para afinar nuestras estimaciones. Como fuente de información complementaria se utilizó el censo industrial de 1965, en el que aparece la composición detallada de todas las sub-ramas industriales, de las cuales se seleccionaron aquellas que producen los principales materiales de construcción que intervienen en la edificación de viviendas, con el propósito de estimar los coeficientes de empleo correspondientes.

Los productos específicos que se requieren para construir una vivienda fueron clasificados en dos grandes sectores:

a) un sector $\boldsymbol{A}$ formado por las sub-ramas seleccionadas dedicadas a la producción de materiales de construcción y que constituyen la mayor parte del consumo intermedio, y en el $A$.

b) un sector $B$, que agrupa a todos los que no se pueden clasificar

Para distinguir a los productos del sector $A$ (compuesto de 16 subramas) se usó el subíndice $k$, y para los del sector $B$ (compuesto de 15 subsectores) el subíndice $i$.

De esta manera, el consumo intermedio en una vivienda, se puede expresar como:

$$
C=\sum_{k} P_{k}+\sum_{i} R_{i}
$$

en donde $P_{k}=$ valor de los productos que tienen como origen la subrama $k$ y $R_{i}=$ valor de los productos que tienen como origen la rama $i$.

Si $y_{k}$ y $z_{i}$, los coeficientes de empleo son conocidos, tendremos definido el empleo indirecto:

$$
E=\sum_{k} y_{k} P_{k}+\sum_{i} z_{i} R_{i}
$$

\section{CÁlculo de los Coeficientes de empleo}

La producción de la sub-rama $k$ puede expresarse:

$$
Q_{k}=\sum_{i} Q_{l k}+\sum_{i} X_{i k}+V_{k}
$$

en donde: $Q_{k}=$ producción bruta de la sub-rama $k$

$Q_{l k}=$ consumo intermedio del producto de la sub-rama $l$ por la sub-rama $k$

$X_{i k}=$ consumo intermedio del producto de la rama $i$ por la sub-rama $k$

$V_{k}=$ valor agregado bruto por la sub-rama $k$ 
Utilizando el mismo cuadro conceptual que el del modelo de Magaud, ${ }^{7}$ podemos establecer:

$$
\begin{gathered}
\alpha_{l k}=Q_{l k} / Q_{k} \\
\beta_{i k}=X_{i k} / Q_{k} \\
y_{k}=\sum_{l} \alpha_{l k} y_{l}+\sum_{i} \beta_{i k} z_{i}+d_{k}
\end{gathered}
$$

en donde $d_{k}$ es el empleo indirecto en la sub-rama $k$.

Con estos elementos se puede obtener el empleo unitario por producto de la sub-rama $k$ sumando el empleo incluido en el consumo del sector $B$ y el empleo directo:

$$
r_{k}=\sum_{i} \beta_{i k} z_{i}
$$

con lo cual tendremos entonces

$$
y_{k}=\sum_{i} \alpha_{l k} y_{l}+d_{k}+r_{k}
$$

o bien

$$
y_{k}=\sum_{l} \gamma_{l k}\left(d_{k}+r_{k}\right)
$$

en donde la matriz $\left[\gamma_{l k}\right]=\left\{I \div\left[\alpha_{l k}\right]\right\}^{-1}$

Ahora bien, dado que no se cuenta con un sistema global que nos permita calcular $d_{k}$, supondremos que la sub-rama tiene el mismo multiplicador que la rama a que pertenece.

Si $M_{k}$ es el empleo en la sub-rama $k$ y $m$ el multiplicador de la rama correspondiente tendremos:

$$
d_{k}=\left(M_{k} / Q_{k}\right) m
$$

\section{Resultados}

Mediante este procedimiento fueron estimados los coeficientes de empleo que aparecen en el cuadro 2, a través de los cuales se puede observar que los productos de "madera semielaborada" y "carpintería" ${ }^{8}$ generan una alta proporción de empleo, lo que se explica por el hecho de que consumen madera, producto del sector primario en cuyas actividades se emplea un gran volumen de mano de obra.

Por su parte, los productos "muebles y materiales de cerámica" y "cemento" generan mano de obra en una proporción muy reducida.

7 Véase Jacques Magaud, op. cit.

8 Véase la clasificación de industrias que aparece en el Censo Industrial. Secretaría de Industria y Comercio, Dirección General de Estadística, México, 1973 
Cuadro 2

México: Coeficientes de EMPleo PaRa 16 sub-Sectores Y TRES CATEGORÍAS a 1965

\begin{tabular}{|c|c|c|c|c|}
\hline & Obreros & Empleados & Profesionistas & Total \\
\hline $\begin{array}{l}\text { Cenento } \\
\text { hrena y grava } \\
\text { Yeso } \\
\text { Cal }\end{array}$ & $\begin{array}{r}8.8 \\
17.3 \\
16.7 \\
21.2\end{array}$ & $\begin{array}{l}1.8 \\
3.2 \\
3.0 \\
3.2\end{array}$ & $\begin{array}{l}0.7 \\
1.9 \\
1.1 \\
1.5\end{array}$ & $\begin{array}{l}19.3 \\
22.4 \\
20.8 \\
25.9\end{array}$ \\
\hline $\begin{array}{l}\text { Pinturas e impermeabilinantes } \\
\text { Madera semi-elaborada } \\
\text { Hierro seni-elaborado }\end{array}$ & $\begin{array}{r}13.0 \\
49.1 \\
9.8\end{array}$ & $\begin{array}{l}4.0 \\
3.4 \\
1.9\end{array}$ & $\begin{array}{l}2.2 \\
1.9 \\
1.1\end{array}$ & $\begin{array}{l}19.2 \\
46.4 \\
72.8\end{array}$ \\
\hline Materiales a base de arcilla & 33.3 & 2.3 & 0.9 & 36.5 \\
\hline $\begin{array}{l}\text { Materiales a base de cenento } \\
\text { Herreria } \\
\text { Vidrierfa } \\
\text { Carpinteria }\end{array}$ & $\begin{array}{r}16.2 \\
19.5 \\
9.9 \\
39.5\end{array}$ & $\begin{array}{l}2.8 \\
2.8 \\
2.6 \\
3.9\end{array}$ & $\begin{array}{l}1.1 \\
1.1 \\
1.1 \\
1.6\end{array}$ & $\begin{array}{l}20.1 \\
23.4 \\
13.6 \\
45.0\end{array}$ \\
\hline Tuehles y acoesorios metálicos & 14.4 & 4.1 & 1.9 & 20.4 \\
\hline Muebles y materiales de cerámíca & 6.9 & 1.5 & 0.7 & 9.1 \\
\hline Materiales y accesorios eléctricos & 13.7 & 2.8 & 1.5 & 18.0 \\
\hline Cerrajerla & 15.5 & 3.6 & 1.5 & 20.6 \\
\hline
\end{tabular}

2 Para una producción de $\$ 1000000$.

\section{Aplicación a un tipo de vivienda}

Una vez estimados los coeficientes de empleo por unidad de producción, para los principales materiales que intervienen en la construcción de viviendas, se procedió a calcular el empleo que se genera en la construcción de una vivienda tipo con especificaciones mínimas, a través de las distintas etapas de la construcción.

En la construcción de vivienda se han distinguido las etapas siguientes:
a) compra de terreno
b) urbanización
c) construcción
d) venta del conjunto

El precio de venta por metro cuadrado para un tipo de vivienda "unifamiliar corriente" y que representa el mínimo que se puede hacer en 1965 para una casa dentro del área metropolitana, aparece en el cuadro 3. El desglose del costo directo aparece en el cuadro 4.

\section{El costo directo}

El costo directo de los insumos de trabajo se obtuvieron mediante la aplicación de los coeficientes ya definidos con los siguientes resul- 


\section{Cuadro 3}

MÉXICo: DESGLOSE DEL PRECIO DE UNA VIVIENDA

\begin{tabular}{|c|c|c|}
\hline Conoep to & . & $\begin{array}{c}\text { Poses por tro tro } \\
\text { oualredo }\end{array}$ \\
\hline Terreno: & & 181.1 \\
\hline $\begin{array}{l}\text { Urbanizaoisn: } \\
\text { Costo direoto } \\
\text { Gastos generales } \\
\text { Impuestos y utilidades }\end{array}$ & & $\begin{array}{r}62.9 \\
48.4 \\
6.3 \\
8.2\end{array}$ \\
\hline $\begin{array}{l}\text { Construocíns } \\
\text { Cos to dirooto } \\
\text { Cestos Beneralos } \\
\text { Impuestos y utilidados }\end{array}$ & $\cdot$ & $\begin{array}{r}\frac{300.6}{231.2} \\
30.1 \\
39.3\end{array}$ \\
\hline $\begin{array}{l}\text { Promocion y vontal } \\
\text { Gas too generales } \\
\text { Impuestos y utilidados }\end{array}$ & & $\begin{array}{l}\frac{65.3}{54.5} \\
10.8\end{array}$ \\
\hline Preoio de venta & & 610.0 \\
\hline
\end{tabular}

Cuadro 4

MÉXICO: ANÁlISIS DEL COSTO DIRECTO DE LA CONSTRUCCIÓN DE LA VIVIENDA TIPO UNIFAMILIAR DE COSTO MÍNIMO

(Porcientos)

\begin{tabular}{|c|c|c|}
\hline & & Ceete direoto \\
\hline 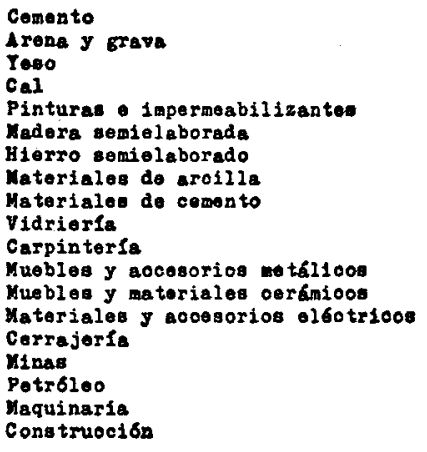 & ! & $\begin{array}{r}3.33 \\
2.58 \\
0.40 \\
0.60 \\
4.15 \\
2.25 \\
13.32 \\
11.22 \\
7.84 \\
0.90 \\
4.53 \\
3.63 \\
2.23 \\
6.31 \\
0.16 \\
2.46 \\
0.02 \\
0.04 \\
1.15\end{array}$ \\
\hline Suma de materiales & & 67.13 \\
\hline $\begin{array}{l}\text { Pebn } \\
\text { oftolal }\end{array}$ & & $\begin{array}{l}13.04 \\
19.83\end{array}$ \\
\hline Suma de mano do obra & & 32.87 \\
\hline Total & & 100.00 \\
\hline
\end{tabular}


tados para cada 1000 metros cuadrados de construcción; expresados en años-hombre:

Empleo directo:

$\begin{array}{ll}\text { Peón } & 3.59 \\ \text { Oficial } & 3.93\end{array}$

Empleo indirecto:

$\begin{array}{ll}\text { Obreros } & 3.02 \\ \text { Empleados } & 0.37 \\ \text { Profesionales } & 0.20\end{array}$

Estos resultados señalan la importancia de la parte no calificada de la mano de obra directa (peones, casi la mitad de los obreros empleados en la obra misma), mientras que en el empleo indirecto, el número de obreros es alrededor de cinco veces mayor que las otras dos categorías (empleados y profesionistas).

Los resultados finales, a partir del costo directo por metro cuadrado, sobre empleo generado por una inversión de $\$ 1000000$ de construcción de viviendas unifamiliares corrientes, son los siguientes (en años-hombre) :

$\begin{array}{lr}\text { Obreros } & 45.5 \\ \text { Empleados } & 1.6 \\ \text { Profesionistas } & 0.9\end{array}$

La distribución del empleo por categoría (obrero, empleado, profesionista) y según su naturaleza (directo, indirecto), en porcientos, es la siguiente:

\begin{tabular}{lcccr}
\hline & Obreros & Empleados & Profesionistas & Total \\
\hline Directo & 67.7 & $\overline{-}$ & $\overline{-}$ & 67.7 \\
Indirecto & 27.2 & 3.1 & 2.0 & 32.3 \\
Total & 94.2 & 3.1 & 2.0 & 100.0 \\
\hline
\end{tabular}

La mano de obra directa absorbe las dos terceras partes del total y la categoría obrero representa casi el $95 \%$ del total, lo que demuestra la importancia de la mano de obra no calificada, o poco calificada, en la construcción de viviendas del tipo considerado.

\section{El precio de venta}

Si se analiza el desglose del precio de venta, el empleo aparece solamente en el costo directo, en gastos generales de urbanización y construcción y en gastos generales de promoción y venta (véase el cuadro 3). En la parte del costo que corresponde a "terreno" se generan en todo caso "ganancias" y no empleo, a pesar que su participación en el precio de venta sea de gran consideración $(30 \%)$. Sin embargo, a pesar de que el costo directo es el elemento más importante en la construcción de viviendas, es necesario considerar el precio total de venta, y medir sus efectos en la generación de empleo. 


\section{Cuadro 5}

EMPLEO INDUCIDO POR 1000 METROS CUADRADOS DE VIVIENDA TIPO UNIFAMILIAR CORRIENTE (Años-hombre)

\begin{tabular}{|c|c|c|c|c|c|c|c|}
\hline & \multicolumn{3}{|c|}{$D i=0 t 0$} & \multicolumn{3}{|c|}{ Indisecto } & \multirow{2}{*}{ Total } \\
\hline & Obreros & Enapleados & Profesionistas & Obreros & Empleados & Profesionistas & \\
\hline $\begin{array}{l}\text { Obra de } \\
\text { construceion }\end{array}$ & 7.52 & $-\because$ & - & 3.02 & 0.37 & 0.20 & 11.11 \\
\hline $\begin{array}{l}\text { Gastos generales } \\
\text { de construceion }\end{array}$ & 0.57 & 0.17 & 0.29 & 0.22 & 0.08 & 0.06 & 1.40 \\
\hline $\begin{array}{l}\text { Obre de } \\
\text { urbanización }\end{array}$ & 1.05 & - & - & 0.60 & 0.09 & 0.05 & 1.80 \\
\hline $\begin{array}{l}\text { Gastos ǧenerales } \\
\text { de urbanizacion }\end{array}$ & 0.12 & 0.04 & 0.06 & 0.05 & 0.02 & 0.01 & 0.29 \\
\hline $\begin{array}{l}\text { Gastos Generales } \\
\text { de formacion y } \\
\text { venta }\end{array}$ & 0.21 & 0.27 & 0.45 & 0.98 & 0.40 & 0.31 & 2.07 \\
\hline Total & 9.47 & 0.27 & 0.45 & 4.87 & 0.97 & 0.63 & 16.67 \\
\hline
\end{tabular}

El efecto total (directo e indirecto) sobre el empleo fue entonces calculado sumando los empleos generados en cada una de las partes mencionadas, obteniendo los resultados que aparecen en el cuadro 5.

Estos resultados se pueden expresar también, a partir del precio por metro cuadrado en empleo generado por una inversión de $\$ 1000000$ en vivienda, en hombres-año:

Empleo directo:

Obreros

Empleados

Profesionistas

0.4

0.7

Empleo indirecto:

$\begin{array}{ll}\text { Obreros } & 8.0 \\ \text { Empleados } & 1.6 \\ \text { Profesionistas } & 1.0\end{array}$

o sea un total de 27.2 hombres-año, para la inversión considerada. La vivienda tipo estudiada genera empleo en menor cantidad que la "construcción" en conjunto. La rama de "construcción" registró un coeficiente de generación de empleo de 29.2 hombres-año.

La distribución del empleo según la categoría y naturaleza del mismo en porcientos es la siguiente:

\begin{tabular}{lcccc}
\hline & Obreros & Empleados & Profesionistas & Total \\
\hline Directo & 56.9 & 1.6 & 2.7 & 61.2 \\
Indirecto & 29.2 & 5.8 & 3.8 & 38.8 \\
Total & 86.1 & 7.4 & 6.5 & 100.0 \\
\hline
\end{tabular}


Estos resultados indican que la parte generada de mano de obra directa se reduce (un poco más de $60 \%$ ) y la parte obrera también (un poco más de $85 \%$ ), lo que se explica con seguridad por el efecto de los gastos generales que incluyen principalmente mano de obra calificada.

Por último, se estimó.que, para la casa promedio considerada (47 metros cuadrados, de costo mínimo), el empleo total generado es de aproximadamente de 0.8 hombres-año, para una vivienda de $\$ 30000$ en 1965 (\$42000.00 en 1970 considerando la variación de precios).

\section{CONClusiones Y RECOMENDACIONES}

Un estudio de esta naturaleza aplicado a distintos tipos de vivienda, permitiría comparar el impacto de cada uno de ellos en la de generación de empleo y serviría para establecer prioridades en los programas de construcción de vivienda desde el punto de vista de la maximización del empleo.

Una aplicación práctica del método podría servir para seleccionar los tipos de vivienda $X_{1}, X_{2}, \ldots X_{m}$ que conviene construir dadas una serie de restricciones (físicas, políticas, económicas, etc.).

Si se conoce el empleo generado por cada tipo de vivienda, el problema podría reducirse a uno de programación lineal en el que se busca maximizar una función dada:

$$
Z=\sum_{j} c_{i} X_{i}
$$

sujeta a las restricciones

$$
\sum_{j} a_{i j} X_{j} \leq b_{i} \quad \mathrm{y}, \quad X_{j} \geq 0
$$

El objetivo "maximizar el empleo" podría ser combinado con otros como el de maximizar las ganancias, maximizar el número de personas atendidas, etc. Pero en tal problema la precisión de los coeficientes de empleo por tipo de vivienda, es esencial a la solución del problema. Pensamos que el método de cálculo expuesto en ese artículo, permite llegar a una buena aproximación, aunque es necesario señalar que se requeriría efectuar un análisis de sensibilidad para comprobar y afinar los resultados. 